\title{
Microbiota of the Gut: Antibiotic-Induced Dysbiosis and the Adverse Effects on Human Health
}

\author{
Matthew TF Lamaudière ${ }^{1}$ and Igor Y Morozov*2 \\ ${ }^{1}$ School of Life Science, Coventry University, UK \\ ${ }^{2}$ Senior Lecturer in Biomolecular Sciences, Centre for Sport, Exercise and Life Sciences, Coventry University, UK
}

*Corresponding author: Igor Y Morozov, Senior Lecturer in Biomolecular Sciences, School of Life Sciences, Coventry University, UK.

Received Date: October 05, 2018

Published Date: October 16, 2018

\begin{abstract}
A healthy gut microbial community is essential for homeostasis in mammals. A symbiotic relationship between host and microbe is essential in developing the immune system, providing biomolecules and generating energy through utilisation of indigestible compounds. The diversity of the gut microbiota is altered following antibiotic treatments, the effect this has on the health and wellbeing of the host has long been underestimated and is now the subject of intense debate. Antibiotics facilitate the selection of energy harvesting microbes within the gut and hence heavily influence the gaining of weight and may be contributing more than we anticipated to the modern obesity epidemic. These changes to the bacterial composition of the gut, dysbiosis are caused by elevated oxygen levels within the gut that promotes the propagation of facultative anaerobic Proteobacteria, a condition associated with inflammation and cancer. Additionally, the altered oxygenated intestinal climate allows the growth of aerobic pathogens, conveying clinically relevant resistance genes on highly transmissible mobile elements between communities or acquiring them from commensal bacteria, in turn aiding the spread of antibiotic resistance. Here we discuss the indirect pleotropic effects antibiotics have on the microbial community and environment of the gut leading to hidden adverse implications to human health.
\end{abstract}

Keywords: Gut microbiota; Anaerobes; Antibiotic treatment; Dysbiosis; Obesity; Inflammation

\section{Introduction}

The intestinal microbiota, one of the densest bacterial communities in mammals, plays an important role in health and wellbeing of the host. The gut microbiota provides the host with essential biomolecules, energy metabolism [1] and has recently been found to be the unexplored epicentre of antibiotic resistance genes [2]. The balanced bacterial community is mainly dominated by obligate anaerobes, Firmicutes and Bacteroidetes due to the strict hypoxic nature of the gut. Obligate anaerobes of the intestine break down indigestible polysaccharides into short-chain fatty acids (SCFA), which are important for the development of the immune system and hence, vital in maintaining homeostasis. Changes in the gut bacterial composition due to e.g. antibiotic $(\mathrm{AB})$ treatments have been shown to lead to both selection of microbes with high energy harvesting capacity thatmay contribute to obesity and an imbalanced microbiota, termed dysbiosis. This is associated with an expansion of facultative anaerobes, Proteobacteria in response to an increase in epithelial oxygenation [3-5]. A shift of the luminal bacterial composition from obligate to facultative anaerobes is associated with inflammation and propagates expansion of pathogens via aerobic respiration [6] as well as commensal Escherichia coli [7]. Potentially, E. coli may carry clinically relevant $\mathrm{AB}$ resistance genes on highly transmissible mobile elements and hence increases the risk of spreading $\mathrm{AB}$ resistance genes [Lamaudiere and Morozov personal communication]. These independent studies suggest a pleotropic effect of $\mathrm{AB}$ on the composition of the human gut with respect to obesity, dysbiosis, infection and the immune system. A more tailored approach to the use of $\mathrm{AB}$ in a clinical setting should be considered, a view that could pave the way to the development of novel preventative initiatives for human gut associated disease.

\section{Discussion}

Anaerobic environment of the healthy mammalian gut governs the bacterial composition and promotes a dominance of obligate anaerobes which constitute around $95 \%$ of the total community 
[8]. They deplete intestinal oxygen via $\beta$-oxidation of microbiotaoriginated butyrate to $\mathrm{CO}_{2}$, the main pathway of energy production, which is vital for the hosts immune system. A disruption of anaerobiosis due to $\mathrm{AB}$ treatment leads to an increased oxygenation of the colon and therefore dysbiosis, an expansion of facultative anaerobic Proteobacteria via aerobic respiration [4,5]. Dysbiosis allows energy to be obtained through anaerobic glycolysis, glucose to lactate [9], that does not require oxygen, leading to increased epithelial oxygenation [10]. This in turn enables an expansion of facultative anaerobes, including commensal and pathogenic E. coli. This can result in a number of consequences as it increases the risk of aerobic pathogens colonizing the gut [6] and predisposes individuals to a number of diseases, including cancer via the activation of pro-inflammatory responses of the immune system $[11,12]$. E. coli constantly produce ethanol, enhancing permeability of the colon. This decreases the defence mechanisms of the host against bacteria-driven toxins such as dimethyl arsine, which is metabolized by $E$. coli to produce micro metabolites, potential carcinogens of the gut [13]. Furthermore, an expansion of $E$. coli in healthy livestock in response to $\mathrm{AB}$ pressure has recently been found to carry mobile elements with highly transmissible resistance determinants. For example, $m r c-1$ and $m c r-2$ alleles confer resistance to last resort $A B$, i.e. colistin and multi-drug efflux pumps, e.g. oqxAB confers resistance to quinolones, $A B$ of the first choice $[14,15]$. This significantly reduces the choice of treatment of infections as well as increasing the risk of spreading resistance within the gut microbiome and beyond. Therefore, $A B$ treatment of infection-free individuals must be considered as high risk, facilitating the emergence and transfer of mobile resistance to human pathogens.

\section{Obesity and dysbiosis}

The common view-point that genetic disposition or dietary habits heavily influence weight gain is contradicted in light of recent data [16]. The enrichment of the microbiome with energy harvesting bacteria elucidates the role of the gut microbiota in the gaining of weight leading to obesity. Recent data has shown that changes in animal gut microbiota in response to $\mathrm{AB}$ treatment resemble the microbiota of obese humans/animals without any dietary alterations [3,17]. Equally, weight gain in humans, both adults and children [18] has been observed with some $A B$ however, effects on health have been disregarded until recently. An $\mathrm{AB}$ dependent selection of both methanogenic Archaea and Prevotellaceae spp together as a novel biomarker of obesity in humans and animals has been recently reported. During the fermentation process by Prevotellaceae the accumulation of excess $\mathrm{H} 2$ reduces the yield of ATP, which leads to a gradual increase in the fermentation process itself [19]. The methanogenic Archaea are believed to not only have a role in obesity through promoting caloric intake from indigestible polysaccharides $[20,21]$, but in addition an increase in Prevotellaceae, can utilise the excess $\mathrm{H} 2$ generated by acetate production, a highly absorbed SCFA [22]. Additionally, Escherichia spp are responsible for increasing intestinal permeability, leading to more efficient absorbance of calories [23]. Furthermore, the animal gut microbiota treated with $A B$ is enriched with Erysipelotrichaceae, bacteria that produce butyrate, another major SCFA source, an excess of which contributes to obesity [24]. Findings strongly argue that the bacterial community of the obese gut is more efficient in extracting calories from food than in a healthier bacterial counterpart and becomes less efficient as the host loses weight, being self-perpetuating through the propagation of bacteria more suited to an obese state. This is consistent with reports that the bacterial gut microbiota of normal mice transplanted into germ-free rodents leads to an increase in the body fat of recipients without any increase in food intake [25]. Hence, $\mathrm{AB}$ should be considered as an important contributor to energy consumption and obesity-preventative programmes should be made an integral part of AB treatment protocol.

\section{Immune disorders}

The symbiosis between the immune system and the anaerobicdominant gut microbiota governs the anaerobic intestinal conditions, being a key determinant in preventing pathogen colonization. Hypo responsive $\mathrm{CD}^{+}{ }^{+} \mathrm{T}$ cells ensure a tolerogenic response to the balanced microbiome via interleukin-10 (IL-10), an anti-inflammatory cytokine, aiding the maintenance of the anaerobic intestinal environment [26]. Consequently, a number of gastrointestinal diseases, e.g. inflammatory bowel disease, which encompasses ulcerative colitis and Crohn's Disease are associated with a pro-inflammatory immune response e.g. IL-17 and IL-23 that sustain an inflammation environment in the tumour [27]. Subsequently, this leads to the selection and propagation on facultative anaerobes, elevating oxygen levels within the gut, dysbiosis through depletion of obligate anaerobes such as Clostridia (phylum Firmicutes), compromising intestinal homeostasis [2830]. The gut microbiota can both protect against and promote the development of Colorectal cancer. The gut Bacteroides fragilis (class Clostridia) are known to induce regulatory T cells (T-reg) and antiinflammatory cytokines. B. fragilis produce polysaccharide A which promotes T-reg development and the clostridial species produce butyrate, the SCFA which stimulates extrathymic differentiation of T-reg and IL-10-producing T-cells that protect against intestinal inflammation [26,31,32]. Additionally, dysbiosis is sufficient to induce tumorigenesis and leads to increased colon permeability, therefore facilitating the access of mutagens $\left(\mathrm{H}_{2} \mathrm{~S}, \mathrm{NO}\right.$, Reactive Oxygen Species and toxins) to colon epithelial cells this, in turn leads to DNA damage [33,34]. Understanding the mechanisms by which the gut microbiota modulates the immune system and hence promotes cancer will lead to the development of novel preventative and therapeutic approaches in stemming intestinal tumorigenesis [35].

\section{Conclusion}

A broad spectrum of human disease, including obesity, intestinal inflammation, infection and cancer appears to be tightly modulated by the composition of microbes dwelling in the gut. A disruption of the hypoxic intestine by environmental cues, e.g. AB or disease, changes the population of the gut microbiota, enabling facultative anaerobes to support and perpetuate luminal dysbiosis. This increases human susceptibility to disease and sustaining a diseased state, hence diminishing effectiveness of treatments. Elucidating the mechanisms by which the gut microbiome modulates human health 
and diseases will mark a paradigm shift in our understanding of the importance of developing personalised preventative medicine. This will include development of fundamentally novel and longterm strategies to control the disease (preventing dysbiosis and the spread of $\mathrm{AB}$ resistance, facilitating weight loss and coordination of the immune system) via manipulating the composition of the gut microbiota.

\section{Acknowledgments}

This work was supported by the NERC grant to IM (NE/ N019288/1). We wish to thank Derek Renshaw for helpful suggestions and critical evaluation of the work.

\section{References}

1. Den Besten G, Van Eunen K, Groen AK, Venema K, Reijngoud DJ et al. (2013) The role of short-chain fatty acids in the interplay between diet, gut microbiota, and host energy metabolism. J Lipid Res 54(9): 23252340 .

2. Allen HK, Donato J, Wang HH, Cloud-Hansen KA, Davies J et al. (2010) Call of the wild: antibiotic resistance genes in natural environments. Nat Rev Microbiol 8(4): 251-259.

3. Looft T, Johnson TA, Allen HK, Bayles DO, Alt DP et al. (2012) In-feed antibiotic effects on the swine intestinal microbiome. Proc Natl Acad Sci U S A 109(5): 1691-1696.

4. Saito K (1961) Studies on the habitation of pathogenic Escherichia coli in the intestinal tract of mice. I. Comparative experiments on the habitation of each type of resistant pathogenic Escherichia coli under an administration of streptomycin Paediatria Japonica. 65: 385-393

5. Vollaard EJ, Clasener HA Janssen AJ (1992) Janssen Co-trimoxazole impairs colonization resistance in healthy volunteers. J Antimicrob Chemother 30(5): 685-691

6. Lopez CA, Miller BM, Rivera-Chavez F, Velazquez EM, Byndloss MX, et al. (2016) Virulence factors enhance Citrobacter rodentium expansion through aerobic respiration. Science 353(6305): 1249-1253.

7. Hughes ER, Winter MG, Duerkop BA, Spiga L, Furtado de Carvalho T, et al. (2017) Microbial respiration and formate oxidation as metabolic signatures of inflammation-associated dysbiosis. Cell Host Microbe 21(2): 208-219.

8. Eckburg PB, Bik EM, Bernstein CN, Purdom E, Dethlefsen L, et al. (2005) Diversity of the human intestinal microbial flora. Science 308(5728): 1635-1638.

9. Donohoe DR, Wali A, Brylawski BP, Bultman SJ. (2012) Microbial regulation of glucose metabolism and cell-cycle progression in mammalian colonocytes. PLoS ONE 7(9): e46589.

10. Kelly CJ, Zheng L, Campbell EL, Saeedi B, Scholz CC, et al. (2015) Crosstalk between microbiota-derived short-chain fatty acids and intestinal epithelial HIF augments tissue barrier function. Cell Host Microbe 17(5): 662-671.

11. Shin NR, Whon TW, Bae JW (2015) Proteobacteria: microbial signature of dysbiosis in gut microbiota. Trends Biotechnol 33(9): 496-503.

12. Litvak Y, Byndloss M X, Tsolis RM, Baumler A J (2017) Dysbiotic Proteobacteria expansion: a microbial signature of epithelial dysfunction. Curr Opin Microbiol 39: 1-6.

13. Yamanaka K, Ohba H, Hasegawa A, Sawamura R, Okada S (1989) Mutagenicity of dimethylated metabolites of inorganic arsenics. Chem Pharm Bull 37(10): 2753-2756.

14. Xavier BB, Lammens C, Ruhal R, Kumar-Singh S, Butaye P, et al. (2016) Identification of a novel plasmid mediated colistin-resistance gene, mcr-2, in Escherichia coli, Belgium. Euro Surveill 21(27): 30280.
15. Hansen LH, Johannesen E, Burmolle M, Sørensen AH, Sørensen SJ (2004) Plasmid-encoded multidrug efflux pump conferring resistance to olaquindox in Escherichia coli. Antimicrob Agents Chemother 48(9): 3332-3337.

16. Ley RE, Turnbaugh PJ, Klein S, Gordon JI (2006) Microbial ecology: human gut microbes associated with obesity. Nature 444(7122): 10221023.

17. Cromwell GL (2002) Why and how antibiotics are used in swine production. Anim Biotechnol 13(1): 7-27.

18. Million M, Lagier JC, Yahav D, Paul M (2013) Gut bacterial microbiota and obesity. Clin Microbiol Inf 19(4): 305-313.

19. Zhang H, DiBaise JK, Zuccolo A, Kudrna D, Braidotti M, et al. (2009) Human gut microbiota in obesity and after gastric bypass. Proc Natl Acad Sci USA 106(7): 2365-2370.

20. Samuel BS, Gordon JI (2006) A humanized gnotobiotic mouse model of host-archaeal-bacterial mutualism. Proc Natl Acad Sci USA 103(26): 10011-10016.

21. Dridi B, Raoult D, Drancourt M (2011) Archaea as emerging organisms in complex human microbiomes. Anaerobe 17(2): 56-63.

22. Chakraborti CK (2015) New-found link between microbiota and obesity. World J Gastrointest Pathophysiol 6(4):110-119.

23. Martinez-Medina M, Denizot J, Dreux N, Robin F, Billard E, et al. (2014) Western diet induces dysbiosis with increased E. coli in CEABAC10 mice, alters host barrier function favouring AIEC colonisation. Gut 63(1): 116-124.

24. Kaakoush NO (2015) Insights into the Role of Erysipelotrichaceae in the Human Host. Front Cell Infect Microbiol 5: 84

25. Bäckhed F, Ding H, Wang T, Hooper LV, Koh GY, et al. (2004) The gut microbiota as an environmental factor that regulates fat storage. Proc Natl Acad Sci USA 101(44):15718-15723.

26. Furusawa Y, Obata Y, Fukuda S, Endo TA, Nakato G, et al. (2013) Commensal microbe-derived butyrate induces the differentiation of colonic regulatory T cells. Nature 504(7480): 446-450.

27. Grivennikov SI, Wang K, Mucida D, Stewart CA, Schnabl B, et al. (2012). Adenomalinked barrier defects and microbial products drive IL-23/IL17-mediated tumour growth. Nature 491(7423): 254-258.

28. Rivera-Chavez F, Zhang LF, Faber F, Lopez CA, Byndloss MX, et al. (2016) Depletion of butyrate-producing clostridia from the gut microbiota drives an aerobic luminal expansion of Salmonella. Cell Host Microbe 19(4): 443-454.

29. Lopez CA, Miller BM, Rivera-Chávez F, Velazquez EM, Byndloss MX, et al. (2016) Virulence factors enhance Citrobacter rodentium expansion through aerobic respiration. Science 353(6305): 1249-1253.

30. Carvalho FA, Koren O, Goodrich JK, Johansson ME, Nalbantoglu I, et al. (2012) Transient inability to manage Proteobacteria promotes chronic gut inflammation in TLR5-deficient mice. Cell Host Microbe 12(2): 139152 .

31. Atarashi K, Tanoue T, Oshima K, Suda W, Nagano Y, et al. (2013) Treg induction by a rationally selected mixture of Clostridia strains from the human microbiota. Nature 500(7461): 232-236.

32. Arpaia N, Campbell C, Fan X, Dikiy S, Van Der Veeken J, et al. (2013) Metabolites produced by commensal bacteria promote peripheral regulatory T-cell generation. Nature 504(7480): 451-455.

34. Cuevas-Ramos G, Petit CR, Marcq I, Boury M, Oswald E, et al. (2010) Escherichia coli induces DNA damage in vivo and triggers genomic instability in mammalian cells. Proc Natl Acad Sci.USA 107(25): 1153711542 .

35. Irrazabal T, Belcheva A, Girardin SE, Martin A., Philpott DL (2014) The Multifaceted Role of the Intestinal Microbiota in Colon Cancer. Mol Cell 54(2): 309-320. 